\title{
circSKIL promotes the ossification of cervical posterior longitudinal ligament by activating the JNK/STAT3 pathway
}

\author{
NAIKUN SUN ${ }^{1,2}$, YUNBANG LIANG ${ }^{2}$, BAOSHAN HU $^{2}$, JINYI FENG $^{2}$, \\ GUANGXUN LIN ${ }^{2}$, XIN CHEN ${ }^{2}$ and GANG RUI ${ }^{1,2}$ \\ ${ }^{1}$ Department of Clinical Medicine, Fujian Medical University, Fuzhou, Fujian 350122; ${ }^{2}$ Department of Orthopedic Surgery, \\ The First Affiliated Hospital of Xiamen University, Xiamen, Fujian 361005, P.R. China
}

Received September 9, 2020; Accepted March 29, 2021

DOI: $10.3892 /$ etm.2021.10193

\begin{abstract}
Ossification of the posterior longitudinal ligament (OPLL) is a hyperostotic spinal condition that involves genetic factors as well as non-genetic factors, and its underlying molecular mechanism is largely unknown. Recently, circular RNAs (circRNAs) have been attracting the attention of researchers since they have important regulatory roles in many diseases, including bone metabolism disorders. The present study aimed to investigate the role of circRNA SKI-like proto-oncogene (circSKIL) in OPLL disease progression. First, primary posterior longitudinal ligament cells from patients with cervical spondylotic myelopathy (CSM) without OPLL (control group) and CSM patients with OPLL (OPLL group) were isolated, and the expression levels of circSKIL in ligament cells was found to be significantly increased in the OPLL group compared with control. This result was also confirmed in OPLL tissues. Next, circSKIL was overexpressed in control ligament cells, and the proliferation, mineralization, and osteogenic differentiation of ligament cells were found to be significantly enhanced; the phosphorylation levels of both JNK and STAT3 were upregulated. By contrast, the knockdown of circSKIL in OPLL ligament cells inhibited proliferation, mineralization, and osteogenic differentiation and inactivated the JNK/STAT3 pathway. Therefore, circSKIL may have a significant role in osteogenic differentiation and could serve as a potential target to prevent OPLL progression.
\end{abstract}

\section{Introduction}

Ossification of the posterior longitudinal ligament (OPLL) is a common musculoskeletal disease, characterized by progressive ectopic ossification in the tissues of the spinal ligament and

Correspondence to: $\mathrm{Dr}$ Gang Rui, Department of Clinical Medicine, Fujian Medical University, 1 Xuefu North Road, Fuzhou, Fujian 350122, P.R. China

E-mail: reigang@163.com

Key words: circular RNA SKI-like protooncogene, posterior longitudinal ligament, ossification, JNK/STAT3 leading to hyperostotic conditions (1-3). Patients with OPLL usually develop various degrees of neurological symptoms, from discomfort to severe myelopathy, due to compression of the spinal cord and nerve roots by the progressively calcified posterior longitudinal ligament (PLL). The development of OPLL depends on multiple factors, including diet, obesity, age, diabetes mellitus, and genetic factors (4); however, the role of fibroblastic cells, the most common cells in connective tissues, including PLL (5), is largely unknown.

Recently, Wang et al (6) analyzed the long non-coding RNAs (lncRNA) in OPLL tissues and identified 143 differentially expressed lncRNAs compared with tissues from healthy controls, which indicated an extensive molecular change in the development of OPLL. The silencing of lncRNA SNHG1, which is highly expressed in OPLL tissues, significantly inhibited the osteogenic differentiation of ligament fibroblastic cells (6). Dickkopf-1 (Dkk1) is a secreted inhibitor of the Wnt pathway, and its expression level was significantly decreased in OPLL patients and ligament cells compared with that in non-OPLL controls (7). Treatment with recombinant Dkk1 markedly reversed the osteogenic differentiation of OPLL ligament cells via inhibition of the Wnt pathway (7). In addition, microRNA (miRNA/miR)-563 levels were found to be significantly higher in OPLL ligament cells, and the silencing of miR-563 considerably inhibited the osteogenic differentiation of OPLL ligament cells (8). These findings indicate that fibroblastic cells from PLL are implicated in the progression of ectopic ossification. Therefore, understanding the underlying molecular mechanism of the osteogenic differentiation of ligament cells is expected to provide a promising therapeutic target for OPLL treatment.

Circular RNAs (circRNAs) are a large endogenous group of non-coding RNAs that form a closed loop structure due to a covalent phosphodiester bond between the $3^{\prime}$ and $5^{\prime}$ ends $(9,10)$. CircRNAs are thought to have a significant role in bone metabolism (11). A previous study has shown that circ19142 and circ5846 are associated with osteoblast differentiation, and that bone morphogenetic protein (BMP) 2 might induce osteogenic differentiation through the circ19142/circ5846-targeted miRNA-mRNA axis (12). CircRNAs hsa_circ_0032462, hsa_circ_0028173, and hsa_circ_0005909 are known to promote cell adhesion molecule 1 expression by functioning as miRNA sponges in human osteosarcoma (13). Additionally, 
the silencing of circIGSF11 promoted osteoblast differentiation and increased the expression of miR-199b-5p in bone marrow stem cells (14). To date, whether circRNA is implicated in the osteoblast differentiation of ligament cells remains unknown.

In the present study, control and OPLL patient ligament samples were collected and the expression levels of circSKIL, which was upregulated in osteogenically differentiated periodontal ligament stem cells (15), were assessed. The role of circSKIL in the proliferation and osteogenic differentiation of ligament cells was analyzed, and the JNK/STAT3 pathway was also examined.

\section{Materials and methods}

Sample collection. Cervical posterior longitudinal ligament tissue samples were collected from 24 patients diagnosed with cervical spondylotic myelopathy (CSM) without OPLL (control group) and from another 24 CSM patients with OPLL (OPLL group) between June 2017 and October 2019. The clinicopathological characteristics of the patients are presented in Table I. The diagnosis of CSM and OPLL was based on the clinical data obtained from computer tomography (CT) and magnetic resonance imaging (MRI). Patients with diffuse idiopathic skeletal hyperostosis or diabetes mellitus were excluded. The modified Japanese Orthopedic Association (mJOA) guidelines were used to evaluate the severity of myelopathy (16), and only patients with moderate symptoms (mJOA score, 12-14) were considered. The OPLL type was diagnosed as segmental, according to a widely used OPLL classification (17). All patients underwent cervical anterior decompression surgery, and the posterior longitudinal ligaments were collected intraoperatively. The current study was approved by the Ethical Committee of the First Affiliated Hospital, Xiamen University (Xiamen, China). The study protocols were in accordance with The Declaration of Helsinki. Informed consent was obtained from all patients prior to sample collection for the study.

Cell culturing of OPLL cells and osteogenic induction. For primary cell isolation, three control ligament tissues and three OPLL tissues from the aforementioned 46 patients were collected and minced to an approximate size of $0.5 \mathrm{~mm}^{3}$. The fragments were washed twice with PBS and plated on a 90-mm culture dish with low-glucose DMEM (Thermo Fisher Scientific, Inc.) supplemented with $10 \%$ fetal bovine serum (FBS; Gibco; Thermo Fisher Scientific, Inc.), 1\% L-glutamine (Gibco; Thermo Fisher Scientific, Inc.) and 1\% penicillin/streptomycin (Gibco; Thermo Fisher Scientific, Inc.). The medium was changed every three days. Passage number 3 cells were used for the experimental studies. For osteogenic differentiation, cells were cultured in differentiation medium, which is an osteogenic inductive DMEM containing various supplements (10\% FBS, $50 \mu \mathrm{g} / \mathrm{ml}$ ascorbate- 2 phosphate, $100 \mathrm{nM}$ dexamethasone, and 10 mM $\beta$-glycerophosphate; Sigma-Aldrich; Merck KGaA). Following 7 or 14 days of osteogenic induction, the cells were used for subsequent assays. All cells were incubated in a humidified atmosphere containing $95 \%$ air and $5 \% \mathrm{CO}_{2}$ at $37^{\circ} \mathrm{C}$.

Alizarin Red $S$ (ARS) staining and quantification. To visualize the mineralization potential of ligament cells, ARS staining was performed, as described previously (18). ARS
Table I. Clinicopathological characteristics of control and OPLL patients.

\begin{tabular}{lcc}
\hline Feature & Control & OPLL \\
\hline Sex & & \\
Male & 14 & 10 \\
Female & 9 & 13 \\
Age & $49.22 \pm 6.43$ & $52.37 \pm 5.28$ \\
Diagnosis & $\mathrm{CSM}$ & CSM and OPLL \\
OPLL type & $\mathrm{NA}$ & segmental \\
mJOA score & $12.55 \pm 0.74$ & $12.75 \pm 0.83$
\end{tabular}

OPLL, ossification of the posterior longitudinal ligament; CSM, cervical spondylotic myelopathy; mJOA, modified Japanese Orthopedic Association.

selectively binds to calcium, yielding a dark red color. Before ARS staining, OPLL cells $\left(1 \times 10^{5}\right.$ cells/well) were cultured in a 12-well plate with osteogenic induction medium for 7 days, as aforementioned. The cultured cells were fixed with 95\% ethanol for $30 \mathrm{~min}$ at room temperature, and then stained with $0.1 \%$ ARS (Sciencell Research Laboratories, Inc.) for $20 \mathrm{~min}$ at room temperature. Images of the calcified matrices were photographed using a conventional camera.

An ARS Staining Quantification Assay kit (Sciencell Research Laboratories, Inc.) was used for the quantification of matrix mineralization. Basically, the stain was eluted using $100 \mathrm{mM}$ cetylpyridinium chloride for $1 \mathrm{~h}$ and spectrophotometric quantification was performed at $562 \mathrm{~nm}$.

Cell proliferation assay. The cell proliferation rate was determined using a CCK-8 assay. Cells were cultured in 96-well plates $\left(3.5 \times 10^{3}\right.$ cells/well) for $24 \mathrm{~h}$ at $37^{\circ} \mathrm{C}$. The cells in various wells overexpressed either circSKIL or the mock plasmid for the control cells group, and either the small interfering (si-) circSKIL or negative control siRNA (si-NC) for the OPLL cells group. Cell proliferation was assessed every day, from the 1st to the 5th day. After each incubation time, $10 \mu \mathrm{l}$ of CCK-8 reagent (Beyotime Institute of Biotechnology) was added to each well and cultured for $1 \mathrm{~h}$ at $37^{\circ} \mathrm{C}$. The absorbance was measured at $450 \mathrm{~nm}$ using a microplate reader. All tests were performed in triplicates.

RNA isolation and reverse transcription-quantitative $P C R$ $(R T-q P C R)$. Total RNA extraction was performed using TRIzol $^{\circledR}$ reagent (Thermo Fisher Scientific, Inc.), according to the manufacturer's instructions. In total, $1 \mu \mathrm{g}$ RNA was reverse transcribed into cDNA using the iScriptcDNA synthesis kit (Bio-Rad Laboratories, Inc.). The reaction was started at $25^{\circ} \mathrm{C}$ for $5 \mathrm{~min}$, followed by $42^{\circ} \mathrm{C}$ for $60 \mathrm{~min}$ and $95^{\circ} \mathrm{C}$ for 5 min. Random primers (Toyobo Life Science) were used for circRNA analysis. ABsolute qPCR SYBR Green Master Mix (Thermo Fisher Scientific, Inc.) was used for qPCR analysis. The total reaction volume $(20 \mu \mathrm{l})$ of buffered solution contained $1 \mu l$ of the diluted (1:5) reverse transcription product and $10 \mathrm{pM}$ sense and antisense primers. Thermocycling was performed as follows: $95^{\circ} \mathrm{C}$ for $5 \mathrm{~min}$, then 42 cycles of $95^{\circ} \mathrm{C}$ for $5 \mathrm{sec}$ and $60^{\circ} \mathrm{C}$ for $1 \mathrm{~min}$. GAPDH was used as the housekeeping gene to 
Table II. Sequences of primers used for reverse transcription-quantitative PCR.

\begin{tabular}{lll}
\hline Gene & \multicolumn{1}{c}{ Forward primer ('5-3') } & \multicolumn{1}{c}{ Reverse primer ('5-3') } \\
\hline circSKIL & AGGAGAAGTTTAGCATGAGAAGTG & AATTGCCCAGTTATCTTCAAGGATT \\
RUNX2 & GGGAACCAAGAAGGCACAGA & GGATGAGGAATGCGCCCTAA \\
ALP & ATCGACGTGATCATGGGTGG & TGGGAATGCTTGTGTCTGGG \\
COL I & GGACACAATGGATTGCAAGGCCGC & TAACCACTGCTCCACTCTGGATGG \\
OCN & ACCTCACAGATGCCAAGCC & GCCGGAGTCTGTTCACTACC \\
OPN & CTCCATTGACTCGAACGACTC & CAGGTCTGCGAAACTTCTTAGAT \\
GAPDH & GTCTCCTCTGACTTCAACAGCG & ACCACCCTGTTGCTGTAGCCAA
\end{tabular}

circ, circular RNA; RUNX2, runt-related transcription factor 2; ALP, alkaline phosphatase; COL I, collagen I; OCN, osteocalcin; OPN, osteopontin.

normalize the expression data. To calculate the fold change in each experiment, the $2^{-\Delta \Delta C q}$ method was used (19). The primers used in the quantitative PCR analysis are listed in Table II. The gene transcription levels of different osteogenic differentiation markers, namely Runt-related transcription factor 2 (RUNX2), alkaline phosphatase (ALP), collagen I (COL I), osteocalcin (OCN), and osteopontin (OPN), were assessed using different primers. The transcription levels of circSKIL were assessed in control cells in which circSKIL was overexpressed and in OPLL cells in which circSKIL expression was downregulated.

Vector construction and cell transfection. To overexpress circSKIL in control cells, the full-length cDNA of circSKIL was amplified and cloned into the pcircRNA 1.2 vector (Guangzhou Boxin Biotechnology Co., Ltd.). The empty plasmid pcircRNA 1.2 without the circSKIL cDNA served as a control vector. To knockdown circSKIL in OPLL cells, siRNAs targeting the back-splice junction of circSKIL (si-circSKIL, 5'-AUCCAAGAUUAAUUAAAAGAA-3') and its negative control (si-NC, 5'-UUCUCCGAACGUGUCACGUTT-3') were designed and synthesized (Guangzhou RiboBio Co., Ltd.).

Cells were seeded in 6-well plates, and $2 \mu \mathrm{g} / \mathrm{ml}$ of plasmids or $50 \mathrm{nM}$ siRNAs were transfected into the corresponding cells using Lipofectamine 2000 (Thermo Fisher Scientific, Inc.) at $37^{\circ} \mathrm{C}$ for $48 \mathrm{~h}$. For the circSKIL overexpression, cells overexpressing plasmids were selected using $300 \mu \mathrm{g} / \mathrm{ml}$ neomycin for 10 days at $37^{\circ} \mathrm{C}$ and confirmed by RT-qPCR, then used for subsequent experiments. For the circSKIL knockdown, expression levels in cells transfected with siRNAs were confirmed by RT-qPCR, and the cells were then used for subsequent experiments.

Western blot analysis. The total protein from the experimental cells was obtained using RIPA lysis buffer (Beyotime Institute of Biotechnology). The protein concentration was determined using a bicinchoninic acid protein assay kit (Biosharp Life Sciences), using bovine serum albumin as a standard. Equal amounts of total protein $(20 \mu \mathrm{g})$ were separated using $12 \%$ sodium dodecyl sulfate polyacrylamide gel electrophoresis (SDS-PAGE). Proteins were transferred onto PVDF membranes (EMD Millipore). After blocking the membranes with 5\% non-fat milk for $1 \mathrm{~h}$ at room temperature, they were incubated with primary antibodies against collagen type I $\alpha 1$ chain $(\mathrm{COL} 1 \mathrm{~A} 1$; cat. no. 39952; 1:1,000; Cell Signaling Technology, Inc.), RUNX2 (cat. no. 12556; 1:1,000; Cell Signaling Technology, Inc.), OCN (cat. no. A18241; 1:1,000; ABclonal Biotech Co., Ltd.), OPN (cat. no. A1361; 1:1,000; ABclonal Biotech Co., Ltd.), ALP (cat. no. A0514; 1:1,000; ABclonal Biotech Co., Ltd.), phosphorylated (p-) JNK (cat. no. 4668; 1:1,000; Cell Signaling Technology, Inc.), JNK (cat. no. 9252; 1:1,000; Cell Signaling Technology, Inc.), p-STAT3 (cat. no. 9145; 1:2,000; Cell Signaling Technology, Inc.), STAT3 (cat. no. 4904; 1:2,000; Cell Signaling Technology, Inc.), and GAPDH (cat. no. 2118; 1:1,000; Cell Signaling Technology, Inc.) at $4^{\circ} \mathrm{C}$ overnight. GAPDH was used as the loading control. After washing with TBST (1\% Tween) twice, the membranes were incubated for $2 \mathrm{~h}$ with HRP-conjugated secondary antibodies (cat. no. 7074; 1:2,000; Cell Signaling Technology, Inc.) at room temperature. The protein signals were detected and visualized using an ECL detection kit (Beyotime Institute of Biotechnology). Densitometry quantification analysis was performed using ImageJ v1.8.0 software (National Institutes of Health).

Statistical Analysis. Quantitative data were expressed as mean \pm standard deviation from at least three independent experiments. All analyses were performed using SPSS 16.0 (SPSS, Inc.). Student's t-test was used to evaluate the statistical significance of differences between two groups. $\mathrm{P}<0.05$ was considered to indicate a statistically significant difference.

\section{Results}

circSKIL is upregulated in OPLL cells and tissues. Primary cervical posterior longitudinal ligament cells were isolated from three patients with CSM without ossification (control) and another three patients with CSM with significant ossification (OPLL), diagnosed according to their MRI and CT data (Fig. 1A). Representative phase-contrast microscopy images from the two groups are presented in Fig. 1B, showing that the cells had a fibroblast-like morphology. The expression levels of circSKIL were detected, and the results indicated that its relative expression levels were significantly higher in the OPLL group compared with the control group (Fig. 1C). For further confirmation, circSKIL expression levels were also detected in 21 tissue samples from patients with OPLL and 21 samples from the control patients, and the results were consistent; 

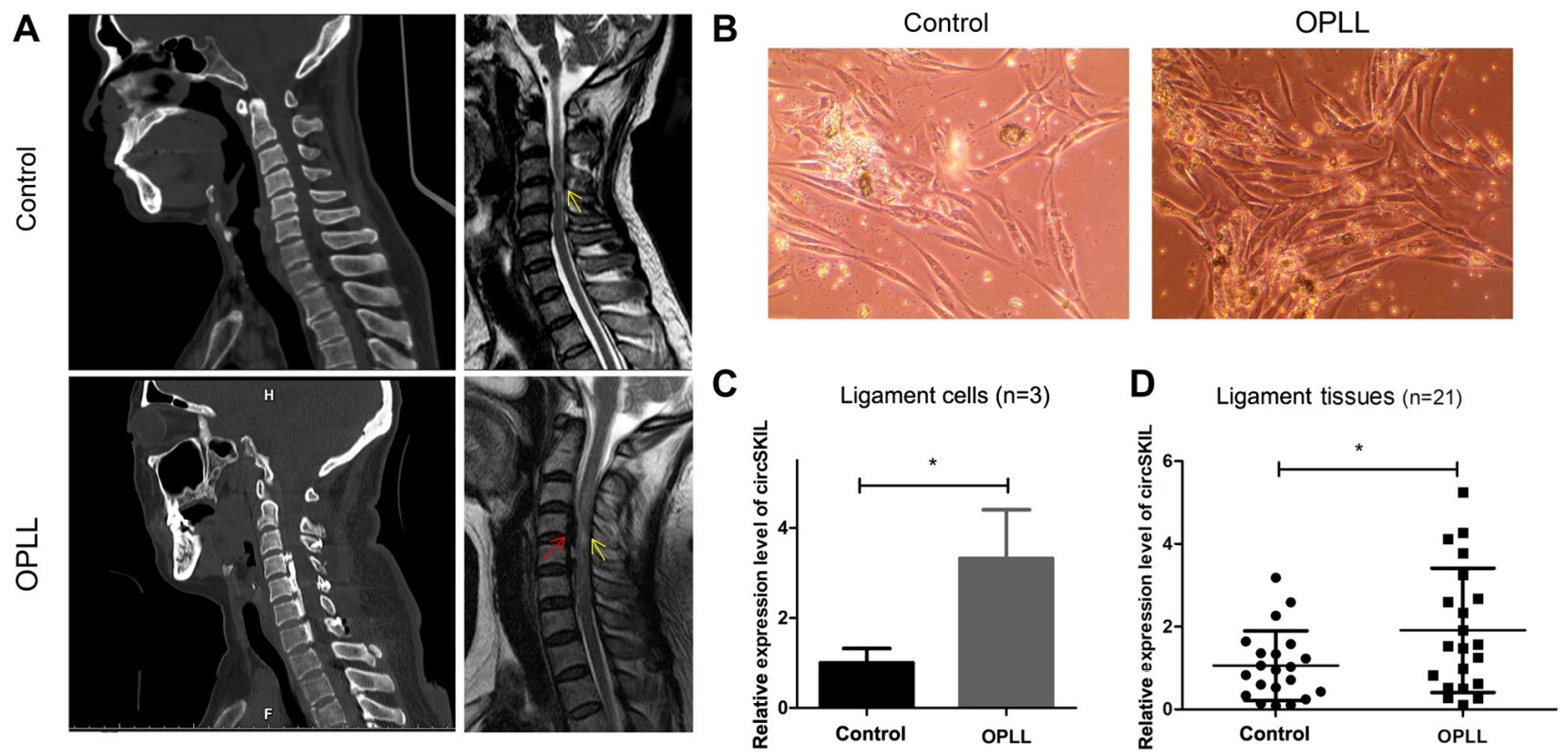

Figure 1. circSKIL is upregulated in OPLL cells and tissues. (A) Representative CT and MRI images of patients in the control and OPLL groups. Red arrow, OPLL lesion; yellow arrow, cervical spondylotic myelopathy lesion. (B) Morphology of primary cervical posterior longitudinal ligament cells. Magnification, x200. (C) circSKIL expression levels in OPLL and control isolated cells. (D) circSKIL expression levels in OPLL and control patient tissues. "P<0.05 vs. control. OPLL, ossification of the posterior longitudinal ligament; circ, circular RNA.

A

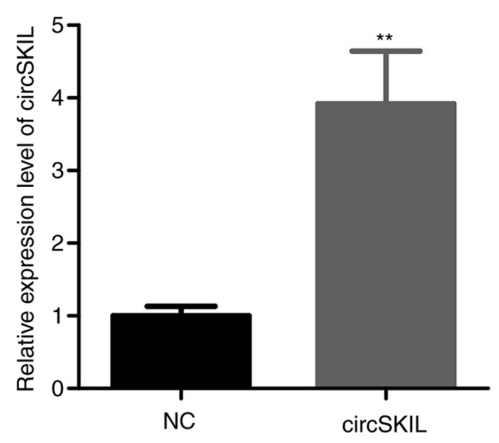

C

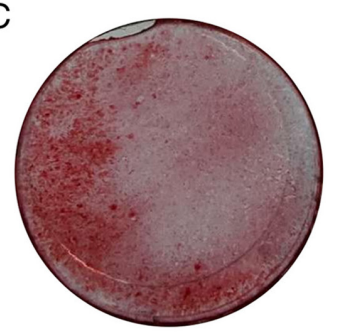

NC

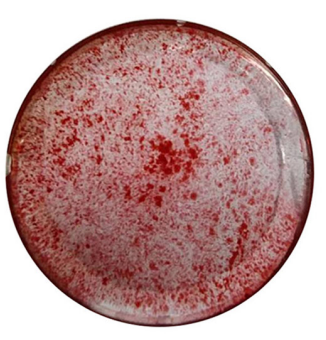

circSKIL

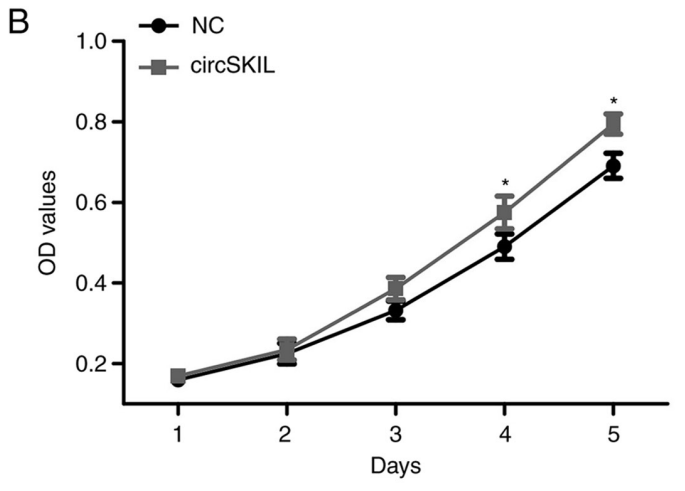

D

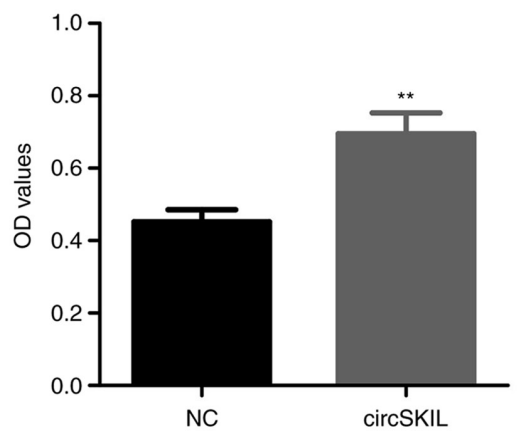

Figure 2. Overexpression of circSKIL promotes the proliferation and mineralization of control cells. Control cells were transfected with either a circSKIL-overexpressing plasmid or an empty vector (NC). (A) Confirmation of successful circSKIL overexpression ( $\mathrm{n}=3$ ). (B) Cell proliferation was assessed by CCK-8 assay ( $n=3)$. (C) Representative images and (D) quantification of Alizarin Red S staining ( $n=3)$. ${ }^{*} \mathrm{P}<0.05$ and ${ }^{* *} \mathrm{P}<0.01$ vs. NC. circ, circular RNA; NC, negative control; OD, optical density.

circSKIL expression levels were significantly elevated in OPLL tissues compared with control tissues (Fig. 1D).

circSKIL overexpression accelerates the proliferation and mineralization of control ligament cells. Next, the function of circSKIL was investigated in control cells by overexpressing circSKIL. Following transfection with an overexpressing plasmid, the levels of circSKIL were significantly higher compared with cells transfected with an empty control vector (NC group; Fig. 2A). The proliferation 

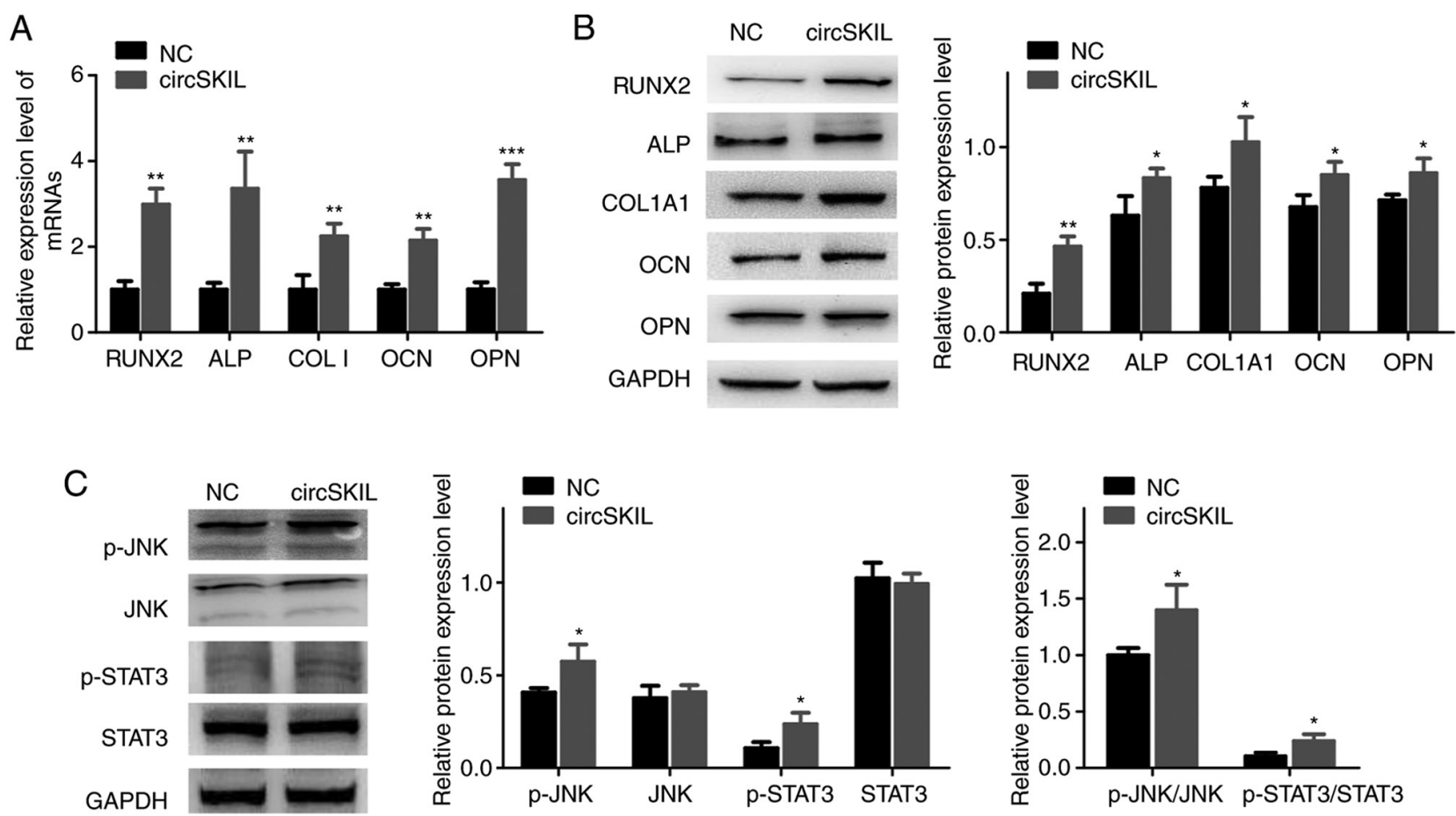

Figure 3. Overexpression of circSKIL promotes the osteogenic differentiation and activates the JNK/STAT3 pathway in control cells. Control cells were transfected with either a circSKIL-overexpressing plasmid or an empty vector (NC). (A) mRNA expression levels of RUNX2, ALP, COL I, OCN, and OPN (n=3). (B) Western blot analysis of the protein expression levels of RUNX2, ALP, COL1A1, OCN, and OPN ( $\mathrm{n}=3$ ). (C) Western blot analysis of the protein levels of p-JNK, JNK, p-STAT3, and STAT3 ( $=3$ ). "P $<0.05,{ }^{* *} \mathrm{P}<0.01$ and ${ }^{* * * *} \mathrm{P}<0.001$ vs. NC. circ, circular RNA; NC, negative control; RUNX2, runt-related transcription factor 2; ALP, alkaline phosphatase; COL I, collagen I; OCN, osteocalcin; OPN, osteopontin; COL1A1, collagen type I $\alpha 1$ chain; p-, phosphorylated.

assay demonstrated a significant increase in the proliferation of circSKIL-overexpressing cells on the 4th and 5th days, compared with that of the NC cells (Fig. 2B). In addition, mineralization was significantly higher in the circSKIL-overexpressing cells compared with that of NC cells, based on ARS staining (Fig. 2C and D).

circSKIL overexpression promotes osteogenic differentiation and activates the JNK/STAT3 pathway in control ligament cells. For further confirmation of osteogenic differentiation, the expression of specific markers was evaluated in control ligament cells overexpressing circSKIL. circSKIL overexpression significantly increased the mRNA expression levels of various osteogenic markers, including RUNX2, ALP, COL I, OCN, and OPN compared with the empty vector-transfected cells (Fig. 3A). Western blot analysis of the circSKIL-overexpressing cells indicated that the protein levels of osteogenic markers were all increased (Fig. 3B), which was consistent with the RT-qPCR results. The JNK/STAT3 pathway was also evaluated, and the results revealed that phosphorylation of both JNK and STAT3 was significantly induced following circSKIL overexpression (Fig. 3C).

circSKIL downregulation inhibits the proliferation and mineralization of OPLL cells. To further investigate the role of circSKIL, its expression was silenced by siRNA in OPLL cells. Following siRNA silencing, the levels of circSKIL were significantly reduced in si-circSKIL cells compared to those in si-NC cells (Fig. 4A). The proliferation assay indicated a significant decrease in proliferation after circSKIL was downregulated in OPLL cells (Fig. 4B). In addition, mineralization in the circSKIL-silenced cells was significantly abrogated compared with that in si-NC cells, based on ARS staining (Fig. 4C and D).

circSKILdownregulation suppresses osteogenic differentiation and inhibits the JNK/STAT3 pathway in OPLL cells. RT-qPCR and western blot results demonstrated that the mRNA and proteins expression levels, respectively, of the different osteogenic markers were significantly reduced in OPLL cells silenced with si-circSKIL compared with si-NC-transfected cells (Fig. 5A and B). In addition, the JNK/STAT3 pathway was significantly inhibited in circSKIL-silenced cells, as evident by a significant decrease in both JNK and STAT3 phosphorylation (Fig. 5C).

\section{Discussion}

OPLL is a challenging spinal disease, with its high incidence affecting the normal life of patients. Anterior and posterior approaches are currently the two major OPLL therapies, but their outcomes remain not satisfactory (20). Multiple factors, such as diet, obesity, age, and diabetes mellitus, are reported to be involved in the progression of OPLL but the underlying molecular mechanism of OPLL is largely unknown.

Emerging evidence has revealed that non-coding RNAs, including lncRNAs and microRNAs (miRNAs), can regulate osteogenic differentiation of ligament cells $(6,8)$; however, the role of circRNA, a rising star in non-coding RNAs, in OPLL progression remains unknown. CircRNA SKIL was 
A

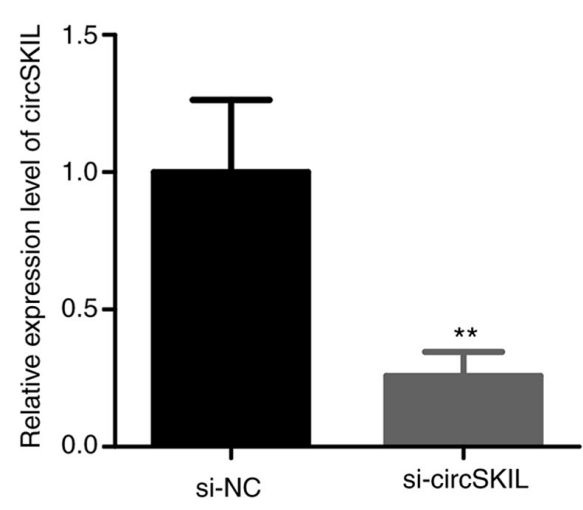

C

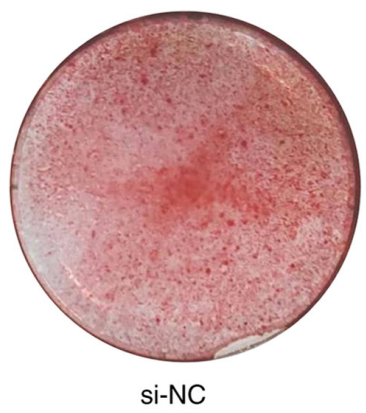

B

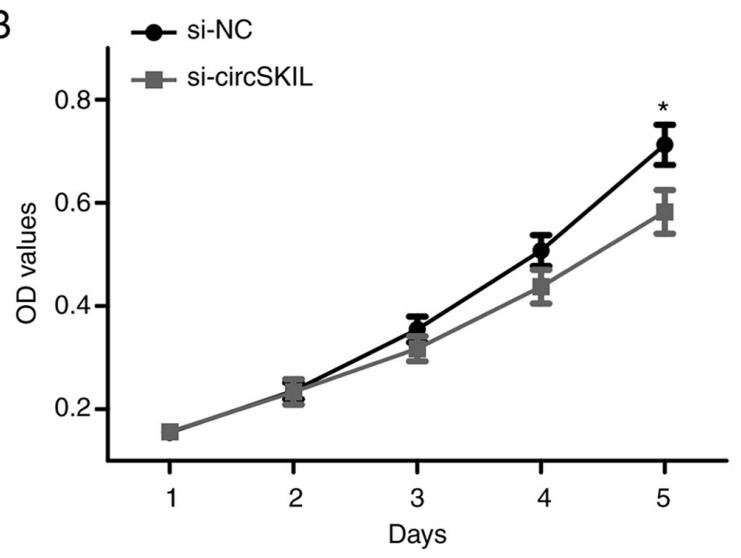

D

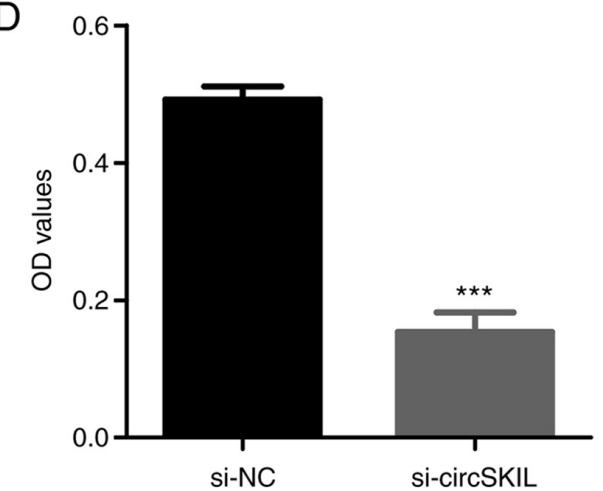

Figure 4. Downregulation of circSKIL inhibits the proliferation and mineralization of OPLL cells. OPLL cells were transfected with either a circSKIL-targeting siRNA or a negative control siRNA (si-NC). (A) Confirmation of successful circSKIL silencing ( $\mathrm{n}=3$ ). (B) Cell proliferation was assessed by CCK-8 assay $(\mathrm{n}=3)$. (C) Representative images and (D) quantification of Alizarin Red $\mathrm{S}$ staining $(\mathrm{n}=3)$. ${ }^{*} \mathrm{P}<0.05,{ }^{* *} \mathrm{P}<0.01$ and ${ }^{* * *} \mathrm{P}<0.001$ vs. si-NC. circ, circular RNA; OPLL, ossification of the posterior longitudinal ligament; si, small interfering; NC, negative control; OD, optical density.

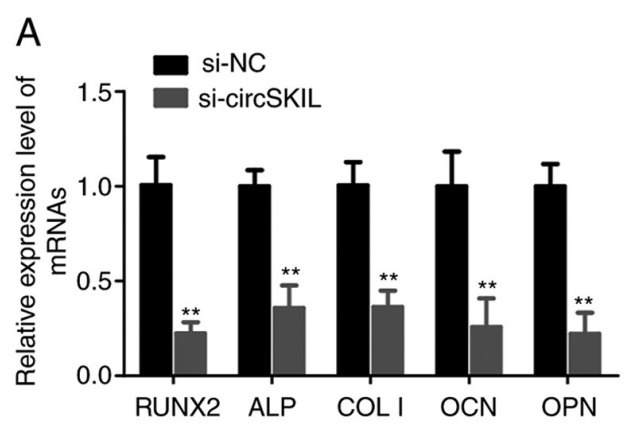

C

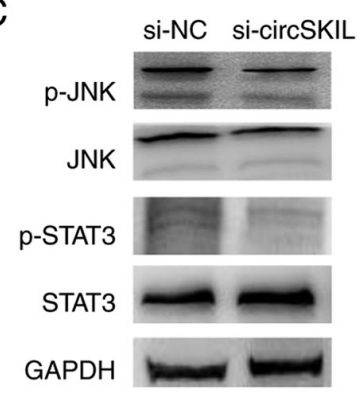

B
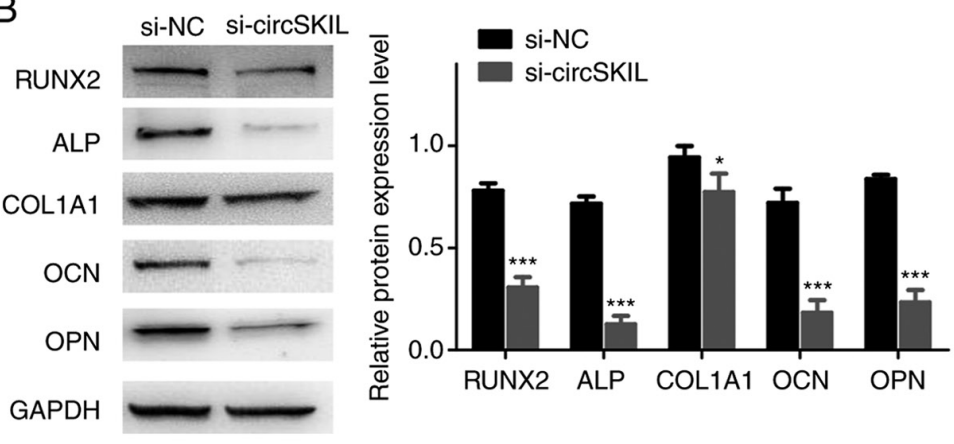

Figure 5. Downregulation of circSKIL suppresses osteogenic differentiation and inhibits the JNK/STAT3 pathway in OPLL cells. OPLL cells were transfected with either a circSKIL-targeting siRNA or a negative control siRNA (si-NC). (A) mRNA expression levels of RUNX2, ALP, COL I, OCN, and OPN (n=3). (B) Western blot analysis of the protein expression levels of RUNX2, ALP, COL1A1, OCN, and OPN ( $\mathrm{n}=3$ ). (C) Western blot analysis of the protein levels of p-JNK, JNK, p-STAT3, and STAT3 ( $\mathrm{n}=3$ ). ${ }^{*} \mathrm{P}<0.05,{ }^{* *} \mathrm{P}<0.01$ and ${ }^{* * * *} \mathrm{P}<0.001$ vs. si-NC. circ, circular RNA; OPLL, ossification of the posterior longitudinal ligament; si, small interfering; NC, negative control; RUNX2, runt-related transcription factor 2; ALP, alkaline phosphatase; COL I, collagen I; OCN, osteocalcin; OPN, osteopontin; COL1A1, collagen type I $\alpha 1$ chain; p-, phosphorylated. 
first identified as upregulated in osteogenically differentiated periodontal ligament stem cells compared with undifferentiated cells using RNA-seq analysis, and its upregulation was confirmed using RT-qPCR (15). The presents study detected its levels in OPLL tissues and cells to explore the expression differences between OPLL and control samples. The present RT-qPCR analyses demonstrated that circSKIL expression levels were significantly increased in OPLL ligament cells and tissues compared with control cells and tissues, respectively, which suggested that an upregulation of circSKIL might play a role in the osteogenic differentiation of ligament cells.

RUNX2 is a key transcription factor that has a crucial role in the early stage of osteoblastic differentiation (21) and is also associated with OPLL (22). ALP is an osteoblast-specific phenotypic marker that is attached to membrane phospholipids of the matrix vesicles and whose activity is increased during the matrix maturation phase (23). COL I is involved in the formation of bone matrix and it is synthesized and secreted by osteoblasts (24). OCN is the most abundant non-collagenous protein in bone matrix and has a crucial role in the process of biomineralization during osteogenic maturation (25). Alterations in the expression levels of these markers are commonly used to identify the osteogenic differentiation level of ligament cells, as previously reported $(6,7,26)$. In the present study, circSKIL was overexpressed in control ligament cells to investigate its role in osteogenic differentiation. The results demonstrated that overexpression of circSKIL significantly induced the mineralization process and increased the expression levels of RUNX2, ALP, COL I, OCN, and OPN. By contrast, the downregulation of circSKIL significantly inhibited the levels of the five osteogenic differentiation markers and the mineralization of OPLL ligament cells.

STAT3 belongs to the signal transducer and activator of transcription family, and the activated STAT3 forms dimers and translocates to the nucleus to regulate the transcription of various genes involved in cellular proliferation and differentiation (27), as well as in osteogenic differentiation (28). The inhibition of STAT3 suppressed osteogenic differentiation-related gene expression in BMSCs induced by hypoxia (29). JNK is an important subfamily of MAPKs, and the activation of JNK has been reported to enhance the osteogenic differentiation of mesodermal progenitor cells (30) and periodontal ligament stem cells (PDLSCs) (31). Hence, the phosphorylation levels of JNK and STAT3 were detected in ligament cells in the present study. The current results indicated that overexpression of circSKIL upregulated p-JNK and p-STAT3 in control ligament cells, whereas silencing of circSKIL inhibited the phosphorylation of the JNK/STAT3 pathway in OPLL cells. Therefore, the role of circSKIL in the osteogenic differentiation of ligament cells may occur via the regulation of the JNK/STAT3 pathway. Inhibition of this pathway may serve as a strategy to alleviate the osteogenic differentiation of ligament cells.

CircRNAs can act as miRNA sponges and regulate the expression of target mRNAs as RNA-induced silencing complexes (10). For example, a circRNA named cerebellar degeneration-related protein 1 transcript (CDR1) promoted the osteogenic differentiation of PDLSCs by acting as an miR-7 inhibitor, which triggers growth differentiation factor 5 upregulation, followed by Smad1/5/8 and p38 MAPK phosphorylation (32). CircPOMT1 and circMCM3AP are known to influence the osteogenic differentiation of human adipose-derived stem cells by targeting hsa-miR-6881-3p via the BMP signaling pathway (33). In addition, several miRNAs, such as miR-204 and miR-211, are able to directly target RUNX2, suppressing its transcriptional activity, thereby inhibiting osteogenic differentiation (34). To date, our knowledge of the role of miRNAs in OPLL is relatively limited compared to other bone disorders, but miRNAs, such as miR-10a (35), miR-1 (36), and miR-17-5p (37), have been reported to inhibit target genes involved in the osteogenesis of OPLL ligament cells. Therefore, several miRNAs might be sponged via circSKIL, thus regulating the osteogenic differentiation of ligament cells, a hypothesis that requires further studies.

A limitation of the present study is that the control samples were not collected from healthy subjects, but from patients with CSM. To exclude the role of CSM on the OPLL mechanism as much as possible, only patients with moderate symptoms were considered for the present study, while those with severe symptoms were excluded.

In conclusion, the present results demonstrated that circSKIL was involved in osteogenic differentiation by regulating the JNK/STAT3 pathway in OPLL ligament cells. The present study provides a novel mechanism for the progression of OPLL.

\section{Acknowledgements}

Not applicable.

\section{Funding}

This work was supported by Xiamen Health and Family Planning Commission (grant no. WKJ2016-2-20).

\section{Availability of data and materials}

The datasets used and/or analyzed during the current study are available from the corresponding author on reasonable request.

\section{Authors' contributions}

GR designed the study. NS, YL and BH performed the experiments and analyzed the data. JF, GL and XC collected and assembled data. GR and NS confirmed the authenticity of the raw data. All authors contributed to the preparation of manuscript and approved the final version.

\section{Ethics approval and consent to participate}

The present study was approved by the Ethics Committee of the First Affiliated Hospital, Xiamen University (Xiamen, China) and written informed consent was obtained from all patients.

\section{Patient consent for publication}

Not applicable. 


\section{Competing interests}

The authors declare that they have no competing interests.

\section{References}

1. Ramos-Remus C, Russell AS, Gomez-Vargas A Hernandez-Chavez A, Maksymowych WP, Gamez-Nava JI, Gonzalez-Lopez L, Garcia-Hernandez A, Meono-Morales E, Burgos-Vargas R and Suarez-Almazor ME: Ossification of the posterior longitudinal ligament in three geographically and genetically different populations of ankylosing spondylitis and other spondyloarthropathies. Ann Rheum Dis 57: 429-433, 1998.

2. Xu N, Yu M, Liu X, Sun C, Chen Z and Liu Z: A systematic review of complications in thoracic spine surgery for ossification of the posterior longitudinal ligament. Eur Spine J 26: 1803-1809, 2017.

3. Guo Q, Ni B, Yang J, Zhu Z and Yang J: Simultaneous ossification of the posterior longitudinal ligament and ossification of the ligamentum flavum causing upper thoracic myelopathy in DISH: Case report and literature review. Eur Spine J 20 (Suppl 2): S195-S201, 2011.

4. Nam DC, Lee HJ, Lee CJ and Hwang SC: Molecular pathophysiology of ossification of the posterior longitudinal ligament (OPLL). Biomol Ther (Seoul) 27: 342-348, 2019.

5. Yu F, Cui Y, Zhou X, Zhang X and Han J: Osteogenic differentiation of human ligament fibroblasts induced by conditioned medium of osteoclast-like cells. Biosci Trends 5: 46-51, 2011.

6. Wang Y, Niu H, Liu Y, Yang H, Zhang $M$ and Wang L: Promoting effect of long non-coding RNA SNHG1 on osteogenic differentiation of fibroblastic cells from the posterior longitudinal ligament by the microRNA-320b/IFNGR1 network. Cell Cycle 19: 2836-2850, 2020.

7. Dong J, Xu X, Zhang Q, Yuan Z and Tan B: Dkk1 acts as a negative regulator in the osteogenic differentiation of the posterior longitudinal ligament cells. Cell Biol Int 44: 2450-2458, 2020.

8. Zhang H, Xu C, Liu Y and Yuan W: MicroRNA-563 promotes the osteogenic differentiation of posterior longitudinal ligament cells by inhibiting SMURF1. Zhonghua Wai Ke Za Zhi 55: 203-207, 2017 (In Chinese).

9. Santosh B, Varshney A and Yadava PK: Non-coding RNAs: Biological functions and applications. Cell Biochem Funct 33: $14-22,2015$

10. Xie L, Huang W, Fang Z, Ding F, Zou F, Ma X, Tao J, Guo J, Xia X, Wang $\mathrm{H}$, et al: CircERCC2 ameliorated intervertebral disc degeneration by regulating mitophagy and apoptosis through miR-182-5p/SIRT1 axis. Cell Death Dis 10: 751, 2019.

11. Patil S, Dang K, Zhao X, Gao Y and Qian A: Role of LncRNAs and CircRNAs in bone metabolism and osteoporosis. Front Genet 11: 584118, 2020

12. Qian DY, Yan GB, Bai B, Chen Y, Zhang SJ, Yao YC and Xia H: Differential circRNA expression profiles during the BMP2-induced osteogenic differentiation of MC3T3-E1 cells. Biomed Pharmacother 90: 492-499, 2017.

13. Chen G, Wang Q, Yang Q, Li Z, Du Z, Ren M, Zhao H, Song Y and Zhang G: Circular RNAs hsa_circ_0032462, hsa_circ_0028173, hsa_circ_0005909 are predicted to promote CADM1 expression by functioning as miRNAs sponge in human osteosarcoma. PLoS One 13: e0202896, 2018.

14. Zhang M, Jia L and Zheng Y: circRNA expression profiles in human bone marrow stem cells undergoing osteoblast differentiation. Stem Cell Rev Rep 15: 126-138, 2019.

15. Gu X, Li M, Jin Y, Liu D and Wei F: Identification and integrated analysis of differentially expressed lncRNAs and circRNAs reveal the potential ceRNA networks during PDLSC osteogenic differentiation. BMC Genet 18: 100, 2017.

16. Fehlings MG, Tetreault LA, Riew KD, Middleton JW, Aarabi B, Arnold PM, Brodke DS, Burns AS, Carette S, Chen R, et al: A clinical practice guideline for the management of patients with degenerative cervical myelopathy: Recommendations for patients with mild, moderate, and severe disease and nonmyelopathic patients with evidence of cord compression. Global Spine J 7 (Suppl 3): 70S-83S, 2017.

17. Tetreault L, Nakashima H, Kato S, Kryshtalskyj M, Nagoshi N, Nouri A, Singh A and Fehlings MG: A systematic review of classification systems for cervical ossification of the posterior longitudinal ligament. Global Spine J 9: 85-103, 2019.
18. Li Y, Wang Y, Li Y, Luo W, Jiang J, Zhao J and Liu C: Controllable synthesis of biomimetic hydroxyapatite nanorods with high osteogenic bioactivity. ACS Biomater Sci Eng 6: 320-328, 2020.

19. Livak KJ and Schmittgen TD: Analysis of relative gene expression data using real-time quantitative PCR and the 2(-Delta Delta C(T)) method. Methods 25: 402-408, 2001.

20. Miao J, Sun J, Shi J, Chen Y and Chen D: A novel anterior revision surgery for the treatment of cervical ossification of posterior longitudinal ligament: Case report and review of the literature. World Neurosurg 113: 212-216, 2018.

21. Franceschi RT, Ge C, Xiao G, Roca H and Jiang D: Transcriptional regulation of osteoblasts. Ann N Y Acad Sci 1116: 196-207, 2007

22. Liu Y, Zhao Y, Chen Y, Shi G and Yuan W: RUNX2 polymorphisms associated with OPLL and OLF in the Han population. Clin Orthop Relat Res 468: 3333-3341, 2010.

23. Jafary F, Hanachi P and Gorjipour K: Osteoblast differentiation on collagen scaffold with immobilized alkaline phosphatase. Int J Organ Transplant Med 8: 195-202, 2017.

24. Wei QS, Huang L, Tan X, Chen ZQ, Chen SM and Deng WM: Serum osteopontin levels in relation to bone mineral density and bone turnover markers in postmenopausal women. Scand J Clin Lab Invest 76: 33-39, 2016.

25. Tsao YT, Huang YJ, Wu HH, Liu YA, Liu YS and Lee OK: Osteocalcin mediates biomineralization during osteogenic maturation in human mesenchymal stromal cells. Int J Mol Sci 18: 159, 2017.

26. Asari T, Furukawa K, Tanaka S, Kudo H, Mizukami H, Ono A, Numasawa T, Kumagai G, Motomura S, Yagihashi S and Toh S: Mesenchymal stem cell isolation and characterization from human spinal ligaments. Biochem Biophys Res Commun 417: 1193-1199, 2012.

27. Ishihara $\mathrm{K}$ and Hirano T: Molecular basis of the cell specificity of cytokine action. Biochim Biophys Acta 1592: 281-296, 2002.

28. Lin J, Cai R, Sun B, Dong J, Zhao Y, Miao Q and Chen C: Gd@ $\mathrm{C} 82(\mathrm{OH}) 22$ harnesses inflammatory regeneration for osteogenesis of mesenchymal stem cells through JNK/STAT3 signaling pathway. J Mater Chem B 6: 5802-5811, 2018.

29. Yu X, Wan Q, Cheng G, Cheng X, Zhang J, Pathak JL and Li Z: $\mathrm{CoCl} 2$, a mimic of hypoxia, enhances bone marrow mesenchymal stem cells migration and osteogenic differentiation via STAT3 signaling pathway. Cell Biol Int 42: 1321-1329, 2018.

30. Matsuguchi T, Chiba N, Bandow K, Kakimoto K, Masuda A and Ohnishi T: JNK activity is essential for Atf4 expression and late-stage osteoblast differentiation. J Bone Miner Res 24: 398-410, 2009.

31. Yang Q, Han Y, Liu P, Huang Y, Li X, Jia L, Zheng Y and Li W: Long noncoding RNA GAS5 promotes osteogenic differentiation of human periodontal ligament stem cells by regulating GDF5 and p38/JNK signaling pathway. Front Pharmacol 11: 701, 2020.

32. Li X, Zheng Y, Zheng Y, Huang Y, Zhang Y, Jia L and Li W: Circular RNA CDR1as regulates osteoblastic differentiation of periodontal ligament stem cells via the miR-7/GDF5/SMAD and p38 MAPK signaling pathway. Stem Cell Res Ther 9: 232, 2018.

33. Huang XQ, Cen X, Sun WT, Xia K, Yu LY, Liu J and Zhao ZH CircPOMT1 and circMCM3AP inhibit osteogenic differentiation of human adipose-derived stem cells by targeting miR-6881-3p. Am J Transl Res 11: 4776-4788, 2019.

34. Huang J, Zhao L, Xing L and Chen D: MicroRNA-204 regulates Runx 2 protein expression and mesenchymal progenitor cell differentiation. Stem Cells 28: 357-364, 2010

35. Xu C, Zhang H, Gu W, Wu H, Chen Y, Zhou W, Sun B, Shen X, Zhang Z, Wang Y, et al: The microRNA-10a/ID3/RUNX2 axis modulates the development of ossification of posterior longitudinal ligament. Sci Rep 8: 9225, 2018.

36. Yuan X, Guo Y, Chen D, Luo Y, Chen D, Miao J and Chen Y: Long non-coding RNA MALAT1 functions as miR-1 sponge to regulate Connexin 43-mediated ossification of the posterior longitudinal ligament. Bone 127: 305-314, 2019.

37. Liao X, Tang D, Yang H, Chen Y, Chen D, Jia L, Yang L and Chen X: Long non-coding RNA XIST may influence cervical ossification of the posterior longitudinal ligament through regulation of miR-17-5P/AHNAK/BMP2 signaling pathway. Calcif Tissue Int 105: 670-680, 2019.

This work is licensed under a Creative Commons Attribution-NonCommercial-NoDerivatives 4.0 International (CC BY-NC-ND 4.0) License. 